\title{
Primordial Non-Gaussianity in Models with Dark Matter Isocurvature Fluctuations
}

\author{
Tomo Takahashi ${ }^{1}$, Masahide Yamaguchi ${ }^{2}$ and Shuichiro Yokoyama ${ }^{3}$ \\ ${ }^{1}$ Department of Physics, Saga University, Saga 840-8502, Japan \\ ${ }^{2}$ Department of Physics and Mathematics, Aoyama Gakuin University, Sagamihara \\ 229-8558, Japan \\ ${ }^{3}$ Department of Physics and Astrophysics, Nagoya University, Aichi 464-8602, Japan
}

\begin{abstract}
We investigate primordial non-Gaussianity and dark matter isocurvature fluctuations in the modulated reheating and the curvaton scenarios. In these scenarios, large non-Gaussianity can be generated, on the other hand, depending on how dark matter is produced, too large isocurvature fluctuations can also arise, which is inconsistent with current observations. In this paper, we study this issue in a mixed scenario where the curvature fluctuations can also be produced from the inflaton fluctuations as well as those from a light scalar field such as the modulus and the curvaton. We show that primordial fluctuations can be highly non-Gaussian without conflicting the current constraint on isocurvature fluctuations for such mixed scenarios. However, if the constraint on isocurvature fluctuations becomes severer as expected by the Planck satellite, $f_{\mathrm{NL}}$, a nonlinearity parameter for adiabatic fluctuations, should be very small as $f_{\mathrm{NL}} \lesssim 3$, which would give interesting implications for the generation mechanism of dark matter. Non-Gaussianity from isocurvature fluctuations is also discussed in these scenarios.
\end{abstract}




\section{Introduction}

Cosmic density fluctuations that we can observe today originate to those generated at the early universe. Since current cosmological observations on density fluctuations are so precise, they provide us a lot of information on the physics of the early universe. Among various observables, non-Gaussianity of primordial perturbations has been attracting much attention recently. One of the reason is that current and upcoming cosmological observations can well probe the Gaussian nature of perturbations more accurately than before. The standard simple inflation model predicts almost Gaussian primordial fluctuations and its deviation from the Gaussian fluctuations is less than $10^{-5}$. Thus, larger deviation from the Gaussian fluctuations, which is still less than $0.1 \%$ of the Gaussian part, would indicate that we need some mechanism of generating primordial curvature perturbations other than the standard single slow-roll field inflation. The size of non-Gaussianity is usually characterized by a non-linearity parameter $f_{\mathrm{NL}}$. The purely Gaussian fluctuations correspond to $f_{\mathrm{NL}}=0$ and the current constraint on this quantity is given as $-9<f_{\mathrm{NL}}<111$ at $95 \%$ C.L. in Ref. [1] and $-4<f_{\mathrm{NL}}<80$ at $95 \%$ C.L. in Ref. [2]\#1.

Another test of primordial fluctuations is the adiabaticity of primordial fluctuations. If any deviation from purely adiabatic fluctuations is discovered, it would have important implications for the generation mechanism of matter (dark matter (DM) and baryon) in the early universe. The adiabatic relation between matter and radiation should be satisfied when both of them are created from a single component. If, however, the deviation from the adiabatic relation is detected, then it implies that radiation and matter have originated from separate components. To quantify the deviation from the adiabaticity, the fraction of the isocurvature fluctuations to the total ones is usually used, which is defined with their power spectra at some reference scale and denoted as $\alpha$. Depending on how isocurvature fluctuations are generated, such an isocurvature mode can be correlated/uncorrelated with the adiabatic ones. The current observational limits on $\alpha_{0}$ (uncorrelated type) and $\alpha_{-1}$ (correlated type) are given by $\alpha_{0}<0.16(0.072)$ at 95\% C.L. from WMAP5-only (from WMAP $5+\mathrm{BAO}+\mathrm{SN})$ and $\alpha_{-1}<0.011(0.0041)$ at 95\% C.L. from WMAP5-only (from $\mathrm{WMAP} 5+\mathrm{BAO}+\mathrm{SN})$, respectively [1].

As a possible mechanism of generating large non-Gaussianity, the curvaton $[3,4]$ and the modulated reheating scenarios [5] have been investigated in various contexts. Although these mechanisms are attractive with regard to producing large non-Gaussianity, when one considers the generation of baryon asymmetry and DM in these mechanisms, large isocurvature fluctuations may arise [6-11], which would indicate that such scenarios are disfavored by cosmological observations.

In our previous letter [11], we have investigated density fluctuations in a scenario with gravitino DM in the framework of modulated reheating [5], which is known to generate large non-Gaussianity [12]. Then, we have shown that gravitino DM is disfavored if the adiabatic curvature perturbations have large local-type non-Gaussianity because of

\#1 Two types of non-Gaussianity are often discussed in the literatures, one is the so-called local type and the other is the equilateral type. In this paper, we only consider the local type non-Gaussianity. 
generating too large DM isocurvature fluctuations simultaneously \#2. However, in general, fluctuations from the inflaton can also contribute to cosmic density fluctuations today even if we consider mechanisms such as the modulated reheating and the curvaton. In such a case, density fluctuations are a mixture of fluctuations originating from multiple sources. Such a mixed scenario in the framework of the modulated reheating [14] and the curvaton [15-20] has been discussed by several authors. With the contribution from the inflaton fluctuations, it is expected that the situation could be dramatically changed because both the isocurvature fluctuations and the non-Gaussianity are suppressed. In this paper, we consider the density fluctuations in a scenario with dark matter isocurvature fluctuations in the mixed modulated reheating and the mixed curvaton scenarios. Then, we investigate whether large non-Gaussianity can be generated without conflicting observational limits on isocurvature fluctuations. Although we focus on the gravitino (axino) DM scenario when we discuss the issue in the framework of the mixed modulated reheating, our discussion on the mixed curvaton scenario can apply to a generic DM which originates from the inflaton or the curvaton. We also discuss non-Gaussianity from isocurvature fluctuations in these scenarios, which can also affect the non-linearity of cosmic microwave background (CMB) temperature fluctuations.

The paper is organized as follows. In section 2, we summarize a formulation to investigate non-Gaussianity based on $\delta N$ formalism and cold dark matter (CDM) isocurvature fluctuations. In section 3, we briefly review the modulated reheating scenario. In section 4, we investigate non-Gaussianity and the gravitino DM isocurvature fluctuations in the mixed modulated reheating scenario. In section 5, we move on to the discussion on the mixed curvaton scenario. In section 6, we discuss the non-linearity of isocurvature fluctuations in these scenarios. Section 7 summarizes our results. Throughout this paper, we set the reduced Planck mass $M_{\mathrm{Pl}}^{2}=(8 \pi G)^{-1}$ to be unity, where $G$ is the gravitational constant.

\section{Formulation}

In this section, we summarize a formalism to discuss primordial non-Gaussianity and isocurvature fluctuations.

\subsection{Non-linearity parameter in $\delta N$ formalism}

First, we give the definition of a non-linearity parameter which characterizes non-Gaussianity of the primordial curvature perturbations. In order to evaluate the curvature perturbations $\zeta$ on super-horizon scales, we adopt the $\delta N$ formalism [21]. In this formalism, the curvature perturbations on sufficiently large scales $\zeta$ at the final time $t=t_{f}$ are identical

\#2 In fact, in the framework of the curvaton scenario [3,4], which is also known as a good candidate to produce large non-Gaussianity [13], such DM scenario may not be viable because of too large isocurvature fluctuations as well. 
to the perturbations of the $e$-folding number measured in the homogeneous FRW Universe from the initial time $t=t_{*}$ to the final time $t_{f}$ as

$$
\zeta\left(t_{f}\right) \simeq \delta N\left(t_{f}, t_{*}\right)
$$

where $N$ represents the $e$-folding number defined as $N\left(t_{f}, t_{*}\right)=\int_{t_{*}}^{t_{f}} H d t$ with $H$ being the Hubble parameter. Usually a final hypersurface at $t=t_{f}$ is taken to be a uniform energy density one and an initial hypersurface at $t=t_{*}$ to be a flat one. Taking the initial time to be some time shortly after horizon crossing during inflation, we can expand $\delta N$ in terms of fluctuations of scalar fields $\varphi^{a}$ on the initial flat hypersurface as

$$
\zeta\left(t_{f}\right) \simeq N_{a} \delta \varphi_{*}^{a}+\frac{1}{2} N_{a b} \delta \varphi_{*}^{a} \delta \varphi_{*}^{b},
$$

up to the second order. Here, a superscript $a$ labels a scalar field and $N_{a} \equiv \partial N\left(t_{f}, t\right) /\left.\partial \varphi^{a}(t)\right|_{t=t_{*}}$ and $N_{a b} \equiv \partial^{2} N\left(t_{f}, t\right) /\left.\partial \varphi^{a}(t) \partial \varphi^{b}(t)\right|_{t=t_{*}}$. The summation is implied for the repeated indices.

To discuss non-Gaussianity, one usually considers the bispectrum (3-point correlation function) of the curvature perturbations which is written as

$$
\left\langle\zeta_{\vec{k}_{1}} \zeta_{\vec{k}_{2}} \zeta_{\vec{k}_{3}}\right\rangle=(2 \pi)^{3} \delta^{(3)}\left(\vec{k}_{1}+\vec{k}_{2}+\vec{k}_{3}\right) B_{\zeta}\left(\vec{k}_{1}, \vec{k}_{2}, \vec{k}_{3}\right)
$$

To quantify the size of non-Gaussianity, the non-linearity parameter $f_{\mathrm{NL}}$ is often adopted and is defined as

$$
B_{\zeta}\left(\vec{k}_{1}, \vec{k}_{2}, \vec{k}_{3}\right)=\frac{6}{5} f_{\mathrm{NL}}\left(P_{\zeta}\left(k_{1}\right) P_{\zeta}\left(k_{2}\right)+2 \text { perms. }\right) .
$$

In the $\delta N$ formalism, we can write the 3 point function of the curvature perturbation as

$$
\begin{aligned}
\left\langle\zeta_{\vec{k}_{1}} \zeta_{\vec{k}_{2}} \zeta_{\vec{k}_{3}}\right\rangle \simeq & (2 \pi)^{3}\left[N^{a} N^{b} N_{a b}+N_{a b} N^{b c} N_{c}^{a} \mathcal{P}_{\delta} \ln \left(k_{m} L\right)\right] \\
& \times\left(P_{\delta}\left(k_{1}\right) P_{\delta}\left(k_{2}\right)+2 \text { perms. }\right) \delta^{(3)}\left(\vec{k}_{1}+\vec{k}_{2}+\vec{k}_{3}\right)
\end{aligned}
$$

where we have neglected the nonlinearity of $\delta \varphi_{*}^{a}$. Here the indices are lowered and raised by using the Kronecker's delta $\delta^{a b}$. The second term in the parenthesis is a contribution from the one-loop correction. $k_{m} \equiv \min \left\{k_{i}\right\}(i=1,2,3)$ and $L$ is a cutoff scale which is often taken to be the order of the present Hubble scale. $P_{\delta}$ and $P_{\zeta}$ represent power spectra of fluctuations of scalar fields and the curvature perturbations, respectively. They are given and related to $\mathcal{P}_{\delta}$ and $\mathcal{P}_{\zeta}$ by

$$
\begin{aligned}
& \left\langle\delta \varphi_{* \vec{k}_{1}}^{a} \delta \varphi_{* \vec{k}_{2}}^{b}\right\rangle \equiv(2 \pi)^{3} \delta^{a b} \delta^{(3)}\left(\vec{k}_{1}+\vec{k}_{2}\right) P_{\delta}\left(k_{1}\right)=\frac{2 \pi^{2}}{k_{1}^{3}} \delta^{a b} \mathcal{P}_{\delta} \delta^{(3)}\left(\vec{k}_{1}+\vec{k}_{2}\right), \\
& \left\langle\zeta_{\vec{k}_{1}} \zeta_{\vec{k}_{2}}\right\rangle \equiv(2 \pi)^{3} \delta^{(3)}\left(\vec{k}_{1}+\vec{k}_{2}\right) P_{\zeta}\left(k_{1}\right)=\frac{2 \pi^{2}}{k_{1}^{3}} \mathcal{P}_{\zeta} \delta^{(3)}\left(\vec{k}_{1}+\vec{k}_{2}\right)
\end{aligned}
$$


The relation between $P_{\zeta}$ and $P_{\delta}$ can be written as

$$
P_{\zeta}(k)=\left[N_{a} N^{a}+N_{a b} N^{a b} \mathcal{P}_{\delta} \ln (k L)\right] P_{\delta}(k) .
$$

Then we can express the non-linearity parameter $f_{\mathrm{NL}}$ as

$$
\frac{6}{5} f_{\mathrm{NL}}=\frac{1}{\left(N_{c} N^{c}+N_{c d} N^{c d} \mathcal{P}_{\delta} \ln \left(k_{m} L\right)\right)^{2}}\left[N^{a} N^{b} N_{a b}+N_{a b} N^{b c} N_{c}^{a} \mathcal{P}_{\delta} \ln \left(k_{m} L\right)\right] .
$$

Here, we neglect the contribution coming from the higher order in $\delta \varphi_{*}^{a}$, for example, terms with $N_{a b c}, N_{a b c d}$, and so on. We will justify this assumption for the modulated reheating scenario latel\#3.

\subsection{Cold dark matter (CDM) isocurvature fluctuations}

Now let us move on to the issue of CDM isocurvature fluctuations. If we consider the curvature perturbations $\zeta_{i}$ on the spatial slices of uniform density $\rho_{i}$ for the $i$-th component, which are related to the total curvature perturbation $\zeta$ as $\zeta=\sum_{i} \zeta_{i} \dot{\rho}_{i} / \dot{\rho}$, isocurvature fluctuations between CDM and radiation are defined as

$$
S_{\mathrm{CDM}} \equiv 3\left(\zeta_{\mathrm{CDM}}-\zeta_{\mathrm{r}}\right)
$$

where $\zeta_{\mathrm{CDM}}$ and $\zeta_{r}$ are the curvature perturbations defined on the slice of $\rho_{\mathrm{CDM}}$ and $\rho_{r}$ being uniform, respectively. One can also write down this quantity using the fluctuations of the ratio between the number density of $\mathrm{CDM}, n_{\mathrm{CDM}}$, and the entropy, $s$, as

$$
S_{\mathrm{CDM}}=\frac{\delta\left(n_{\mathrm{CDM}} / s\right)}{n_{\mathrm{CDM}} / s}=\frac{\delta n_{\mathrm{CDM}}}{n_{\mathrm{CDM}}}-\frac{\delta s}{s} .
$$

To parametrize the contribution from isocurvature fluctuations, one usually uses the fraction of isocurvature fluctuations to the total ones which is defined as

$$
\alpha \equiv \frac{P_{S}\left(k_{0}\right)}{P_{\zeta}\left(k_{0}\right)+P_{S}\left(k_{0}\right)},
$$

where $k_{0}$ is some reference scale at which the power spectra are evaluated. $P_{S}(k)$ is the power spectrum for isocurvature fluctuations defined by

$$
\left\langle S_{\vec{k}_{1}} S_{\vec{k}_{2}}\right\rangle \equiv(2 \pi)^{3} \delta^{(3)}\left(\vec{k}_{1}+\vec{k}_{2}\right) P_{S}\left(k_{1}\right)=\frac{2 \pi^{2}}{k_{1}^{3}} \mathcal{P}_{S} \delta^{(3)}\left(\vec{k}_{1}+\vec{k}_{2}\right) .
$$

In the scenario discussed in the following, isocurvature fluctuations can be correlated/uncorrelated with adiabatic ones and both can arise simultaneously. In such a case, we need to define

${ }^{\# 3}$ For the curvaton scenario, as shown in Ref. [22], one can find that the third order terms with $N_{a b c}$ can be neglected for large $f_{\mathrm{NL}}$ in the absence of the non-linear evolution of the curvaton field between the horizon crossing and the start of curvaton oscillation. 
the fraction $\alpha$ separately for correlated and uncorrelated ones, respectively. Detailed discussion on this point will be made in the next section.

As in the case for the curvature (adiabatic) fluctuations, we can also define the nonlinearity parameters for isocurvature fluctuations $f_{\mathrm{NL}}^{(\mathrm{iso})}$ as

$$
S_{\mathrm{CDM}}=S_{g}+f_{\mathrm{NL}}^{(\mathrm{iso})} S_{g}^{2}
$$

where $S_{g}$ is the Gaussian part of isocurvature fluctuations. In some cases, the contribution from the second order term dominates over the first order one. In such a case, the fraction of isocurvature fluctuations $\alpha$ represents the size of non-Gaussianity. Regarding the notation for non-Gaussianity from isocurvature fluctuations, we follow those of Ref. [23].

\section{Modulated reheating scenario}

In this section we give a brief review of the modulated reheating scenario and some results for non-Gaussianity of the curvature perturbations and gravitino DM isocurvature fluctuations in this scenario.

\subsection{Non-Gaussianity of the curvature perturbations}

Let us start with considering the background dynamics during reheating era, in order to evaluate the curvature perturbations in the modulated reheating scenario based on $\delta N$ formalism. Here we assume that the inflaton oscillates under a quadratic potential after inflation and hence the energy density of the inflaton behaves like a matter during its oscillation. The homogeneous background equations during reheating era are given by

$$
\begin{aligned}
& \frac{d \rho_{\phi}}{d N}+3 \rho_{\phi}=-\frac{\Gamma}{H} \rho_{\phi}, \\
& \frac{d \rho_{r}}{d N}+4 \rho_{r}=\frac{\Gamma}{H} \rho_{\phi}, \\
& H^{2}=\frac{1}{3}\left(\rho_{\phi}+\rho_{r}\right),
\end{aligned}
$$

where $\rho_{\phi}$ and $\rho_{r}$ are energy densities of the inflaton and radiation, respectively. $\Gamma$ is the decay rate of the inflaton into radiation. In the modulated reheating scenario, the decay rate of the inflaton depends on a light scalar field (so-called modulus) $\sigma$. Thus $\Gamma$ can fluctuate due to fluctuations of the modulus. The $e$-folding number $N$ during reheating era can be given by

$$
N\left(t_{f}, t_{i}\right)=\ln \left(\frac{a\left(t_{f}\right)}{a\left(t_{i}\right)}\right),
$$

where $a(t)$ is the scale factor. The initial time $t_{i}$ and the final time $t_{f}$ are respectively taken to be the time at the end of inflation and some time after the completion of reheating. 
Under the sudden decay approximation, the inflaton decays into the radiation at $H=\Gamma$, suddenly. Then, one can rewrite the $e$-folding number as

$$
N\left(t_{f}, t_{i}\right)=\ln \left(\frac{a\left(t_{\mathrm{dec}}\right)}{a\left(t_{i}\right)}\right)+\ln \left(\frac{a\left(t_{f}\right)}{a\left(t_{\mathrm{dec}}\right)}\right),
$$

where $t=t_{\mathrm{dec}}$ is the time when $H=\Gamma$. From $t=t_{i}$ to $t=t_{\mathrm{dec}}$, the Universe is dominated by the inflaton behaving like matter and then becomes dominated by radiation after $t=t_{\mathrm{dec}}$. Hence, we have

$$
N\left(t_{f}, t_{i}\right)=-\frac{2}{3} \ln \left(\frac{\Gamma}{H\left(t_{i}\right)}\right)-\frac{1}{2} \ln \left(\frac{H\left(t_{f}\right)}{\Gamma}\right),
$$

where we have used $H \propto a^{-3 / 2}$ during matter dominated era and $H \propto a^{-2}$ during radiation dominated era. In the $\delta N$ formalism, the curvature perturbations can be generated from fluctuations of the decay rate originating from those fluctuations of the modulus $\delta \sigma_{*}$,

$$
\zeta\left(t_{f}\right) \simeq \delta N=N_{\sigma} \delta \sigma_{*}+\frac{1}{2} N_{\sigma \sigma} \delta \sigma_{*}^{2}
$$

where

$$
\begin{aligned}
& N_{\sigma}=\frac{\partial N}{\partial \Gamma} \Gamma^{\prime}(\sigma) \\
& N_{\sigma \sigma}=\frac{\partial N}{\partial \Gamma} \Gamma^{\prime \prime}(\sigma)+\frac{\partial^{2} N}{\partial \Gamma^{2}}\left(\Gamma^{\prime}(\sigma)\right)^{2}
\end{aligned}
$$

From Eq. (20), we find

$$
\begin{aligned}
& \frac{\partial N}{\partial \Gamma}=-\frac{1}{6} \frac{1}{\Gamma}, \\
& \frac{\partial^{2} N}{\partial \Gamma^{2}}=\frac{1}{6} \frac{1}{\Gamma^{2}} .
\end{aligned}
$$

Using these expressions, we can evaluate the power spectrum of the curvature perturbations and also the non-linearity parameter $f_{\mathrm{NL}}$ in the modulated reheating scenario given by Eq. (9).

\subsection{Gravitino DM isocurvature fluctuations}

Here we discuss isocurvature fluctuations from gravitino DM in the modulated reheating scenarid\#4. There are two major ways to produce gravitino DM\#5. One is from the

\footnotetext{
\#4 The same discussion also applies to axino DM which are produced by thermal scattering during reheating [24].

\#5 Gravitinos can also be produced by the decay of the next-to-the-lightest supersymmetric particle (NLSP). However, the detailed calculations show that the constraints from BBN are severer than those from the overclosure of the universe, irrespective of the kind of particle of NLSP as long as it is the MSSM particle [25]. Therefore, a scenario with (most) DM being gravitinos can be realized only for the following two cases.
} 
scattering of particles in the thermal plasma and their relic abundance is evaluated as [26],

$$
\frac{n_{3 / 2}}{s} \simeq 10^{-12} \sum_{i} g_{i}^{2}\left(1+\frac{m_{G i}^{2}}{3 m_{3 / 2}^{2}}\right)\left(\frac{T_{R}}{10^{10} \mathrm{GeV}}\right)
$$

where $T_{R}$ is the reheating temperature, $m_{3 / 2}$ is the gravitino mass, $m_{G i}$ is the gaugino masses for $i$-th generation, and $g_{i}$ is the gauge coupling. Notice that the relic abundance is proportional to the reheating temperature $T_{R}$. The other way is to produce gravitinos non-thermally from the decay of some heavy scalar field such as the inflaton or moduli [27]. In this case, the yield can be written as

$$
\frac{n_{3 / 2}}{s}=\frac{3}{2} B_{3 / 2} \frac{T_{R}}{m_{3 / 2}}
$$

where $B_{3 / 2}$ is the branching ratio of the decay into gravitinos. When gravitinos are produced from the jets, $B_{3 / 2}$ should be understood as those including its multiplicity. Since the reheating temperature is related to the decay rate of the inflaton as $\Gamma \propto T_{R}^{2}$, then $B_{3 / 2} \propto T_{R}^{-2}$, we have the $T_{R}$-dependence of the $n_{3 / 2} / s$, in the case of non-thermal production, as

$$
\frac{n_{3 / 2}}{s} \propto \frac{1}{T_{R}} .
$$

In the modulated reheating scenario, the reheating temperature fluctuates in space due to the fluctuations of the modulus field $\sigma$, thus gravitino DM isocurvature fluctuations can be generated as

$$
S_{3 / 2}=\frac{\delta\left(n_{3 / 2} / s\right)}{n_{3 / 2} / s}= \pm \frac{\delta T_{R}}{T_{R}},
$$

where the positive and negative signs correspond to the cases with thermal and nonthermal productions, respectively. Hence, we can easily find that if the gravitinos constitute DM in the Universe, isocurvature fluctuations are generated in both cases.

From current observations such as $\mathrm{CMB}$, the magnitude of isocurvature fluctuations is strongly constrained. Since $T_{R}$ is proportional to $\Gamma^{1 / 2}$, we find $\delta T_{R} / T_{R}=\delta \Gamma /(2 \Gamma)$. Then using the curvature perturbations given by Eq. (21), $S_{3 / 2}$ can be related to $\zeta$ as

$$
S_{3 / 2}= \pm \frac{\delta T_{R}}{T_{R}}= \pm \frac{\delta \Gamma}{2 \Gamma} \simeq \mp 3 \zeta
$$

Thus we have $S_{3 / 2} / \zeta \simeq \mp 3$, which is fully correlated to the curvature perturbations and already contradicts with the current observations [11]. In fact, too large isocurvature fluctuations can also be generated when we consider the production of gravitino DM in the curvaton mechanism. Since the modulated reheating and the curvaton scenarios are the major mechanisms of generating large non-Gaussianity, if the primordial curvature fluctuations are found to be highly non-Gaussian in the future, gravitino DM scenarios may be disfavored because of too large isocurvature fluctuations [11]. However, when the 
curvature fluctuations from the inflaton also contribute to today's density fluctuations, which we call a mixed scenario, the fraction of isocurvature fluctuations would be diluted. Thus in such a case, the DM scenario discussed above may be liberated. On the other hand, non-Gaussianity, which can be generated from fluctuations of the modulus or curvaton, would also be reduced. Thus it is interesting to investigate how large non-Gaussianity can be generated without conflicting the constraint on isocurvature fluctuations in such mixed scenarios of the modulated reheating and the curvaton. In the next section, we discuss the mixed scenario of the modulated reheating. Then in Section 5, we consider such a scenario in the framework of the curvaton.

\section{Mixed modulated reheating scenario}

In this section, we discuss the gravitino DM isocurvature fluctuations and non-Gaussianity in the mixed modulated reheating scenario where fluctuations of the inflaton also contribute to the curvature perturbations as well as those from the modulated reheating.

\subsection{Non-Gaussianity}

In the $\delta N$ formalism, the curvature perturbations in the mixed case are, up to the second order, given by

$$
\zeta=\zeta_{(\phi)}+\zeta_{(\sigma)}
$$

where $\zeta_{(\phi)}$ and $\zeta_{(\sigma)}$ represent the curvature perturbations originating from the inflaton and another scalar field which is assumed to be the modulus here, respectively. They are written as

$$
\begin{aligned}
\zeta_{(\phi)} & \equiv N_{\phi} \delta \phi_{*}, \\
\zeta_{(\sigma)} & \equiv N_{\sigma} \delta \sigma_{*}+\frac{1}{2} N_{\sigma \sigma} \delta \sigma_{*}^{2} .
\end{aligned}
$$

Here we have neglected the non-linearity coming from the inflaton fluctuations since it is of the order of slow-roll parameters and very small. Notice that $\zeta_{(\phi)}$ or $\zeta_{(\sigma)}$ is different from $\zeta_{\phi}$ or $\zeta_{\sigma}$, which is often seen in the literatures. The former represents the contribution to the total curvature perturbation coming from the fluctuations of $\phi$ or $\sigma$ while the latter is the curvature perturbation on the slices of uniform density $\rho_{\phi}$ or $\rho_{\sigma}$. Then, the power spectrum is given by

$$
P_{\zeta}(k)=P_{\phi}(k)+P_{\sigma}(k)
$$

where $P_{\phi}$ and $P_{\sigma}$ are power spectra which are defined by

$$
\begin{aligned}
\left\langle\zeta_{(\phi)}(\vec{k}) \zeta_{(\phi)}\left(\vec{k}^{\prime}\right)\right\rangle & =(2 \pi)^{3} \delta^{(3)}\left(\vec{k}+\vec{k}^{\prime}\right) P_{\phi}(k), \\
\left\langle\zeta_{(\sigma)}(\vec{k}) \zeta_{(\sigma)}\left(\vec{k}^{\prime}\right)\right\rangle & =(2 \pi)^{3} \delta^{(3)}\left(\vec{k}+\vec{k}^{\prime}\right) P_{\sigma}(k) .
\end{aligned}
$$


Here we have assumed that $\phi$ and $\sigma$ are uncorrelated.

For the discussion later, we define the ratio of the power spectra between $P_{\sigma}$ and $P_{\phi}$ at some reference scale $k_{0}$ and express them as

$$
R \equiv \frac{P_{\sigma}}{P_{\phi}}=\frac{N_{\sigma}^{2}}{N_{\phi}^{2}}\left[1+\frac{N_{\sigma \sigma}^{2}}{N_{\sigma}^{2}} \mathcal{P}_{\delta} \ln (k L)\right],
$$

with

$$
\begin{aligned}
N_{\phi}^{2} & =\left(\frac{V}{V^{\prime}}\right)^{2}=\frac{1}{2 \epsilon}, \\
N_{\sigma}^{2} & =\frac{1}{36}\left(\frac{\Gamma^{\prime}}{\Gamma}\right)^{2}, \\
\frac{N_{\sigma \sigma}}{N_{\sigma}^{2}} & =6\left[1-\frac{\Gamma^{\prime \prime} / \Gamma}{\left(\Gamma^{\prime} / \Gamma\right)^{2}}\right] .
\end{aligned}
$$

Here for simplicity we have considered the standard slow-roll inflation model and $\epsilon$ is socalled a slow-roll parameter. With this definition, the limits of $R \rightarrow 0$ and $\infty$ correspond to the pure inflaton and pure modulus cases, respectively. By using $R$, the non-linearity parameter $f_{\mathrm{NL}}$ given by Eq. (9) can be rewritten as

$$
\frac{6}{5} f_{\mathrm{NL}}=\frac{R}{(1+R)^{2}} \frac{N_{\sigma \sigma}}{N_{\phi}^{2}} .
$$

In principle, the term with $N_{\sigma \sigma \sigma}$ can also arise in $R_{\# 6}$. However, by a simple inspection of such a term, we can see that such higher order derivative terms can be neglected. If we take into account the contribution from the third order term in $\delta \sigma, R$ can be expressed as

$$
R=\frac{N_{\sigma}^{2}}{N_{\phi}^{2}}\left[1+\left(\frac{N_{\sigma \sigma}^{2}}{N_{\sigma}^{2}}+\frac{N_{\sigma \sigma \sigma}}{N_{\sigma}}\right) \mathcal{P}_{\delta} \ln \left(k_{m} L\right)\right]
$$

Now we compare $N_{\sigma \sigma}^{2} / N_{\sigma}^{2}$ with $N_{\sigma \sigma \sigma} / N_{\sigma}$ and show that the latter is much smaller than the former, in particular, when $f_{\mathrm{NL}}$ is large. From Eqs. (39) and (40), we obtain

$$
N_{\sigma \sigma \sigma} \simeq-\frac{1}{6}\left[2\left(\frac{\Gamma^{\prime}}{\Gamma}\right)^{3}-3\left(\frac{\Gamma^{\prime}}{\Gamma}\right)\left(\frac{\Gamma^{\prime \prime}}{\Gamma}\right)\right] .
$$

Thus the ratio of these combinations is given by

$$
\frac{N_{\sigma \sigma \sigma} / N_{\sigma}}{N_{\sigma \sigma}^{2} / N_{\sigma}^{2}}=\frac{2-3 \frac{\Gamma^{\prime \prime} / \Gamma}{\left(\Gamma^{\prime} / \Gamma\right)^{2}}}{\left[1-\frac{\Gamma^{\prime \prime} / \Gamma}{\left(\Gamma^{\prime} / \Gamma\right)^{2}}\right]^{2}} .
$$

\footnotetext{
\#6 In fact, the term with $\Gamma^{\prime \prime \prime}=d^{3} \Gamma(\sigma) / d \sigma^{3}$ can also appear. However, in an explicit model we consider in the following, such higher order derivatives vanish. Thus we neglect such a term.
} 
From Eq. (41) we can easily find that at least we need $N_{\sigma \sigma} / N_{\sigma}^{2} \gg 1$ in order to realize large $f_{\mathrm{NL}}$ and then this condition corresponds to

$$
\left|\frac{\Gamma^{\prime \prime} / \Gamma}{\left(\Gamma^{\prime} / \Gamma\right)^{2}}\right| \gg 1 \text {. }
$$

Under this condition Eq. (44) is approximately given by

$$
\left|\frac{N_{\sigma \sigma \sigma} / N_{\sigma}}{N_{\sigma \sigma}^{2} / N_{\sigma}^{2}}\right| \sim\left[\frac{\Gamma^{\prime \prime} / \Gamma}{\left(\Gamma^{\prime} / \Gamma\right)^{2}}\right]^{-1} \ll 1 .
$$

Hence as far as we consider the case with $f_{\mathrm{NL}} \gg \mathcal{O}(1)$, the term with $N_{\sigma \sigma \sigma}$ in the one-loop correction is negligible.

Similarly, we can easily confirm that higher order terms like $N_{\sigma \sigma \sigma \sigma}$ and $N_{\sigma \sigma \sigma \sigma \sigma}$, which can arise in the one-loop correction term of bispectrum and trispectrum, are also negligible.

\subsection{Gravitino DM isocurvature fluctuations}

Here, we discuss the gravitino DM isocurvature fluctuations in the mixed modulated reheating scenario. Gravitino DM isocurvature fluctuations are given by

$$
\begin{aligned}
S_{3 / 2} & =\frac{1}{2} \frac{\Gamma^{\prime}}{\Gamma} \delta \sigma_{*}+\frac{1}{2}\left[\frac{1}{2} \frac{\Gamma^{\prime \prime}}{\Gamma}-\frac{1}{4}\left(\frac{\Gamma^{\prime}}{\Gamma}\right)^{2}\right] \delta \sigma_{*}^{2} \\
& =-3\left[N_{\sigma} \delta \sigma_{*}+\frac{1}{2} N_{\sigma \sigma}\left(1-3 \frac{N_{\sigma}^{2}}{N_{\sigma \sigma}}\right) \delta \sigma_{*}^{2}\right],
\end{aligned}
$$

which are correlated with the adiabatic fluctuations as $S_{3 / 2} \simeq-3 \zeta_{\sigma}$ as discussed in the previous section\#7. Since here we consider a mixed scenario where the curvature fluctuations can also be generated from the inflaton, only some fraction of the isocurvature perturbations is correlated with the (total) curvature perturbations. Then, $S_{3 / 2}$ can be divided into the following two parts,

$$
\begin{aligned}
S_{3 / 2} & =S_{\text {corr }}+S_{\text {uncorr }}, \\
S_{\text {corr }} & =-3 \frac{P_{\sigma}}{P_{\zeta}}\left(\zeta_{(\phi)}+\zeta_{(\sigma)}\right)=-\frac{3 R}{1+R} \zeta, \\
S_{\text {uncorr }} & =\frac{3 R}{1+R}\left(\zeta_{(\phi)}-\frac{P_{\phi}}{P_{\sigma}} \zeta_{(\sigma)}\right)=\frac{3}{1+R}\left(R \zeta_{(\phi)}-\zeta_{(\sigma)}\right),
\end{aligned}
$$

\footnotetext{
${ }^{\# 7}$ In fact, the relation between the isocurvature and adiabatic fluctuations slightly deviates from $S_{3 / 2}=$ $-3 \zeta_{\sigma}$ due to the non-linear terms. However, in order to realize large non-Gaussianity, the condition given by Eq. (45) must be satisfied. Hence, by neglecting $N_{\sigma}^{2} / N_{\sigma \sigma}$ in the non-linear term of $S_{3 / 2}$ we approximately obtain $S_{3 / 2} \simeq-3 \zeta_{\sigma}$.
} 
where the first part is denoted as $S_{\text {corr }}$ and the second as $S_{\text {uncorr }}$. Using the expression (37), we have the following relations,

$$
P_{S_{\text {corr }}}=\frac{R}{1+R} P_{S_{3 / 2}}, P_{S_{\text {uncorr }}}=\frac{1}{1+R} P_{S_{3 / 2}} .
$$

To express the size of the contribution from isocurvature fluctuations, we define the ratio of the power spectrum relative to the total one as

$$
\begin{array}{r}
\alpha_{\text {corr }} \equiv \frac{P_{S_{\mathrm{corr}}}}{P_{\zeta}+P_{S_{\mathrm{corr}}}}, \\
\alpha_{\text {uncorr }} \equiv \frac{P_{S_{\text {uncorr }}}}{P_{\zeta}+P_{S_{\text {uncorr }}}},
\end{array}
$$

for the correlated and uncorrelated parts, respectively. Following the notation of Ref. [1], we define the cross-correlation coefficient $\beta$ as

$$
\beta \equiv-\frac{P_{S_{\mathrm{corr}} \zeta}}{\sqrt{P_{\zeta} P_{S_{\mathrm{corr}}}}},
$$

where $P_{S_{\text {corr } \zeta}}$ denotes the cross-correlation power spectrum defined by

$$
\left\langle S_{\mathrm{corr} \vec{k}_{1}} \zeta_{\vec{k}_{2}}\right\rangle \equiv(2 \pi)^{3} \delta^{(3)}\left(\vec{k}_{1}+\vec{k}_{2}\right) P_{S_{\mathrm{corr} \zeta}}\left(k_{1}\right)
$$

Hence the correlation coefficient is $\beta=+1(-1)$ for the thermally (non-thermally) produced gravitino DM in the modulated reheating scenario.

As already mentioned, the size of isocurvature fluctuations is now severely constrained by observations of CMB and so on. In fact, in the present model, correlated and uncorrelated isocurvature perturbations coexist, thus we need to take into account both contributions simultaneously to obtain observational constraints on $\alpha_{\text {corr }}$ and $\alpha_{\text {uncorr }}$. However, such analysis is not available in the literatures. Hence as reference values, we adopt the constraints on $\alpha_{\text {corr }}$ and $\alpha_{\text {uncorr }}$ obtained separately from recent WMAP5 results: $\alpha_{\text {corr }}<0.011$ and $\alpha_{\text {uncorr }}<0.16$ at $95 \%$ C.L. from the WMAP-only analysis $[1] \# 8$.

\subsection{A Simple Model}

Now let us work on some explicit model of the modulated reheating scenario. We consider the following interaction between an inflaton $\phi$ and a fermion $\psi$ :

$$
\mathcal{L}_{\text {int }} \sim-g(\sigma) \phi \bar{\psi} \psi
$$

\footnotetext{
\#8 In fact, the constraint for correlated isocurvature fluctuations here is obtained for $\beta=-1$. However, it is expected that the sign of the correlation does not affect the constraint on the size much. Thus we refer this value regardless of the sign of $\beta$ as a reference value.
} 
where the coupling constant $g$ depends on a modulus field $\sigma$. We assume that $g(\sigma)$ can be written as

$$
g(\sigma) \simeq g_{0}\left[1+g_{1}\left(\frac{\sigma}{M}\right)+g_{2}\left(\frac{\sigma}{M}\right)^{2}\right]
$$

where $M$ is some energy scale, $g_{1}$ and $g_{2}$ are some coefficients and $|\sigma| / M<1$ is assumed. The decay rate of the inflaton through this interaction is given by

$$
\Gamma \sim \frac{g^{2}}{8 \pi} m_{\phi}
$$

which implies that the form of the decay rate of inflaton is

$$
\Gamma=\Gamma_{0}\left[1+A\left(\frac{\sigma}{M}\right)+B\left(\frac{\sigma}{M}\right)^{2}\right],
$$

where $A$ and $B$ are some coefficients. Substituting Eq. (57) to Eqs. (39) and (40), we obtain

$$
N_{\sigma}^{2}=\frac{1}{36 M^{2}}\left[\frac{A+2 B(\sigma / M)}{1+A(\sigma / M)+B(\sigma / M)^{2}}\right]^{2}
$$

and

$$
N_{\sigma \sigma}=\frac{1}{6 M^{2}}\left[\left(\frac{A+2 B(\sigma / M)}{1+A(\sigma / M)+B(\sigma / M)^{2}}\right)^{2}-\frac{2 B}{1+A(\sigma / M)+B(\sigma / M)^{2}}\right] .
$$

Now we discuss the non-linearity parameter $f_{\mathrm{NL}}$ and the size of isocurvature fluctuations in this model. There are four parameters in the model; $M, \sigma / M, A$ and $B$. Here, we will show the results for $f_{\mathrm{NL}}$ and the isocurvature fraction by considering the limit of $|\sigma| / M \ll 1$ or $A \rightarrow 0$.

In the case of $|\sigma| / M \ll 1, N_{\sigma}^{2}$ in Eq. (58) and $N_{\sigma \sigma}$ in Eq. (159) are respectively reduced as

$$
N_{\sigma}^{2} \simeq\left(\frac{A}{6 M}\right)^{2}, \quad N_{\sigma \sigma} \simeq \frac{1}{6 M^{2}}\left(A^{2}-2 B\right)
$$

On the other hand, for the limit of $A \rightarrow 0$, these are written as

$$
N_{\sigma}^{2} \simeq \frac{1}{36 M^{2}}\left[\frac{2 B(\sigma / M)}{1+B(\sigma / M)^{2}}\right]^{2}, \quad N_{\sigma \sigma} \simeq \frac{1}{6 M^{2}}\left[\left(\frac{2 B(\sigma / M)}{1+B(\sigma / M)^{2}}\right)^{2}-\frac{2 B}{1+B(\sigma / M)^{2}}\right] .
$$

In Fig. 1, we show contours of $f_{\mathrm{NL}}$ along with $\alpha_{\text {corr }}$ (left panel) and $\alpha_{\text {uncorr }}$ (right panel) in the $M-A$ plane. We have fixed the value of $B$ as $B=-1.0$ and assumed $|\sigma| / M \ll 1$. In Fig. 2, we show the same but in the $M-|B|$ plane. In the figure, we have fixed as $A=1.0$ and assumed $|\sigma| / M \ll 1$. In Fig. 3 , we have fixed the value of model parameters 

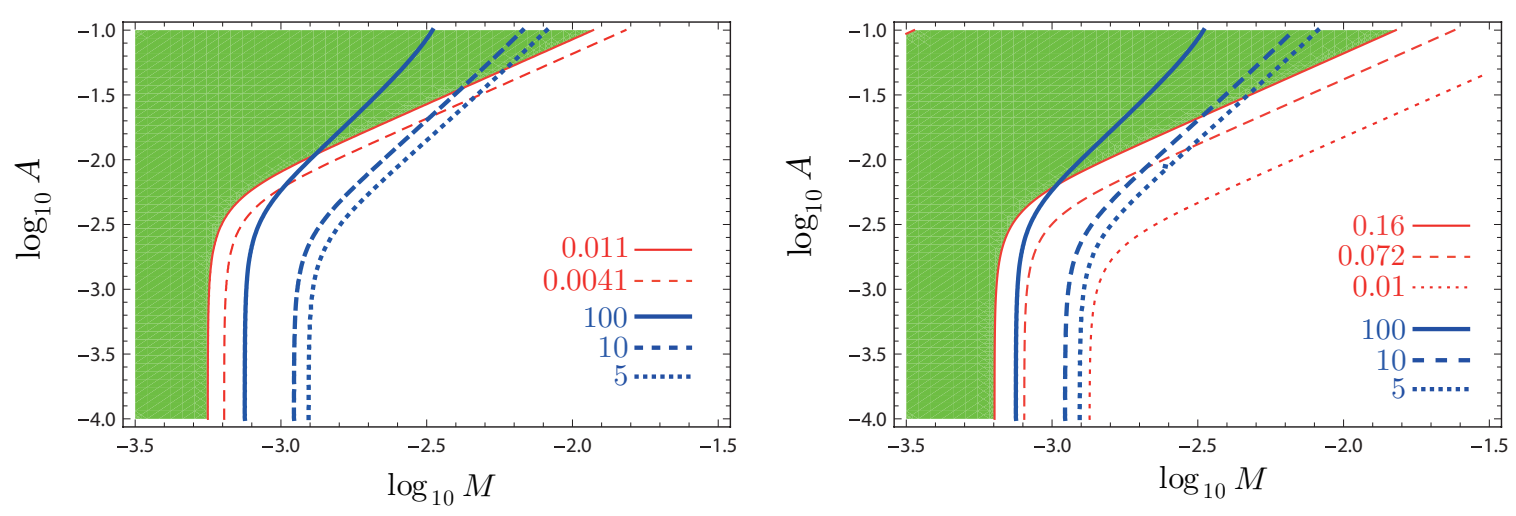

Figure 1: (color online) In the left (right) panel, contours of $f_{\mathrm{NL}}$ and $\alpha_{\text {corr }}\left(\alpha_{\text {uncorr }}\right)$ in the $M-A$ plane are shown. We have fixed as $B=-1.0$ and assumed $|\sigma| / M \ll 1$. Red thin lines in the left panel show contours of $\alpha_{\text {corr }}=0.011$ (solid line) and 0.0041 (dashed line). In the right panel, the lines are shown for $\alpha_{\text {uncorr }}=0.16$ (solid line), 0.072 (dashed line) and 0.01(dotted line). Green shaded regions are constrained by the current observational limit for the isocurvature fractions from WMAP5-only analysis. Blue thick lines show contours of $f_{\mathrm{NL}}=5$ (dotted line), 10 (dashed line) and 100 (solid line).
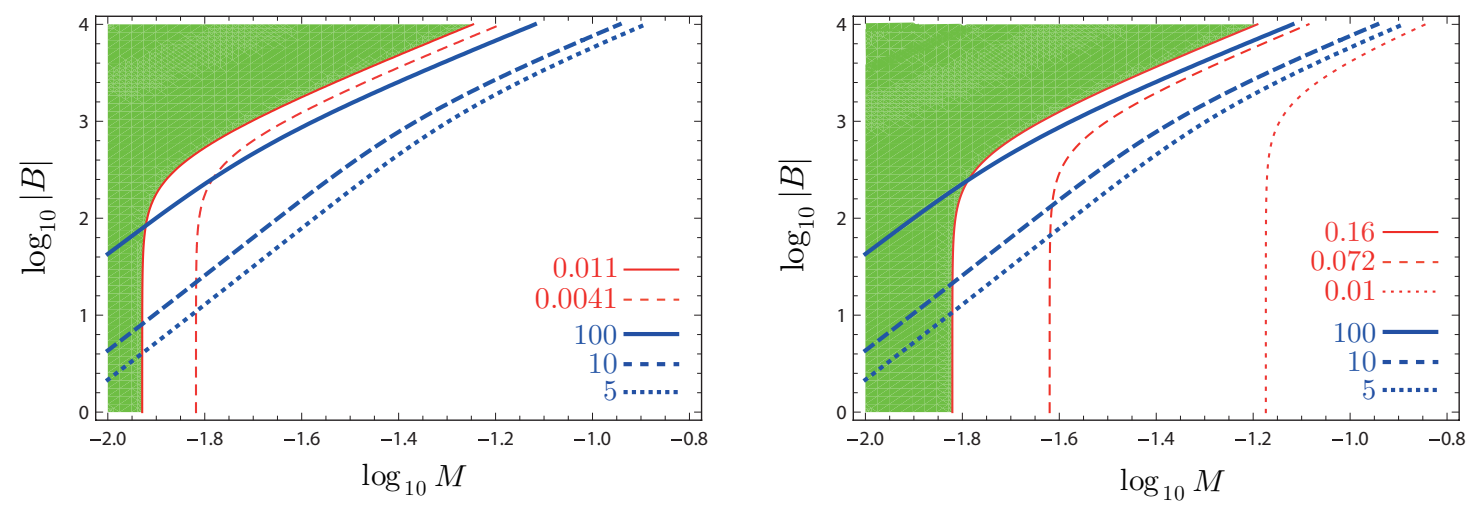

Figure 2: (color online) In the left (right) panel, contours of $f_{\mathrm{NL}}$ and $\alpha_{\text {corr }}\left(\alpha_{\text {uncorr }}\right)$ in the $M-|B|$ plane are shown. We have fixed as $A=1.0$ and assumed $|\sigma| / M \ll 1$. 

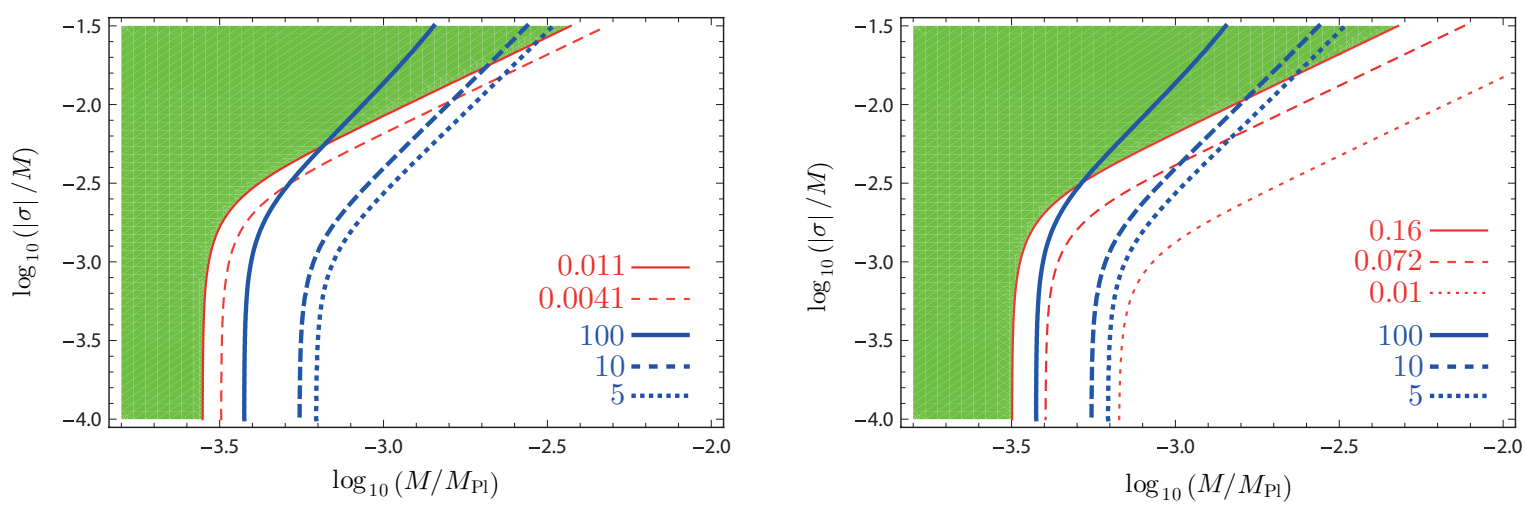

Figure 3: (color online) In the left (right) panel, contours of $f_{\mathrm{NL}}$ and $\alpha_{\text {corr }}\left(\alpha_{\text {uncorr }}\right)$ in the $M-|\sigma| / M$ plane are shown. We have fixed as $B=-1.0$ and $A=0$.

as $B=-1.0$ and $A=0$, then show contours of $f_{\mathrm{NL}}$ as well as $\alpha_{\text {corr }}$ and $\alpha_{\text {uncorr }}$ in the $M-|\sigma| / M$ plane. Regarding the slow-roll parameter $\epsilon$ for the inflaton, we take $\epsilon=0.01$ in the following analyses. In all figures, red thin lines in the left panel correspond to contours of $\alpha_{\text {corr }}=0.011$ (solid line) and 0.0041 (dashed line). These numbers correspond to the $95 \%$ C.L. limit from WMAP5 only and WMAP 5+BAO+SN analyses, respectively. In the right panel, they correspond to contours of $\alpha_{\text {uncorr }}=0.16$ (solid line) and 0.072 (dashed line). These numbers again correspond to the $95 \%$ C.L. limit from WMAP5 only and WMAP5+BAO+SN analyses, respectively. In the near future, we will have a more stringent limit from PLANCK [28-30] and its projected limit on uncorrelated isocurvature fluctuations will be $\alpha_{\text {uncorr }}<0.01$, thus we also show the contour of $\alpha_{\text {uncorr }}=0.01$ with dotted line. Green shaded regions are constrained by the current observational limit for the isocurvature fractions from WMAP-only analysis. Blue thick lines show contours of $f_{\mathrm{NL}}=5$ (dotted line), 10 (dashed line) and 100 (solid line).

From these figures, we can see that large values of $f_{\mathrm{NL}} \sim(10-100)$ can still be realized without conflicting the current constraint on isocurvature fluctuations. In particular, when the one-loop correction term dominates, the following simple relation holds between $f_{\mathrm{NL}}$ and $\alpha_{\text {uncorr }}$ :

$$
f_{\mathrm{NL}} \simeq 3 \times\left(\frac{\alpha_{\text {uncorr }}}{0.01}\right)^{3 / 2}
$$

In Figs. 11 and 3, the regions where the one-loop term dominates correspond to downside of the figures and in Fig. 2, it corresponds to upside of the figure. In these parameter regions, the present limit on the isocurvature fluctuations still allows relatively large nonGaussianity in the scenario.

Here it should be mentioned that the isocurvature constraint is severer for uncorrelated one. The reason is as follows. Here we consider the case where the adiabatic curvature perturbations are mainly generated from fluctuations of the inflaton field, which corresponds 
to the case with $R \ll 1$. On the other hand, the gravitino DM isocurvature fluctuations are produced from fluctuations of the modulus field as given in Eq. (47). Hence, in the small $R$ region, the gravitino DM isocurvature fluctuations have mostly become uncorrelated type. This fact can be also understood by noting Eq. (49). In the near future, the data from PLANCK will be available and the constraint on isocurvature fluctuations would be much severer than that of the current one. The projected limit on uncorrelated isocurvature fluctuations would be $\alpha_{\text {uncorr }}<0.01$ at $95 \%$ C.L. [28]. Thus, for reference, we also plot the corresponding contour in the figures, from which we can find that, if we obtain such a stringent constraint, large non-Gaussianity cannot be generated even in the mixed scenario. Thus if in the future, large local-type non-Gaussianity is confirmed, but the isocurvature constraint becomes as severe as that mentioned above, gravitino DM scenario would be ruled out even if we consider the mixed scenario.

\subsection{Brief comments on the trispectrum}

Before closing this section, we would like to make brief comments on the trispectrum in the mixed modulated reheating scenario. The local type trispectrum can be parameterized with two non-linearity parameters $g_{\mathrm{NL}}$ and $\tau_{\mathrm{NL}}$ as [31]

$$
\begin{aligned}
\left\langle\zeta_{\vec{k}_{1}} \zeta_{\vec{k}_{2}} \zeta_{\vec{k}_{3}} \zeta_{\vec{k}_{4}}\right\rangle \equiv(2 \pi)^{3}[ & \tau_{N L}\left(P_{\zeta}\left(k_{13}\right) P_{\zeta}\left(k_{3}\right) P_{\zeta}\left(k_{4}\right)+11 \text { perms. }\right) \\
& \left.+\frac{54}{25} g_{N L}\left(P_{\zeta}\left(k_{2}\right) P_{\zeta}\left(k_{3}\right) P_{\zeta}\left(k_{4}\right)+3 \text { perms. }\right)\right] \delta^{(3)}\left(\vec{k}_{1}+\vec{k}_{2}+\vec{k}_{3}+\vec{k}_{4}\right)
\end{aligned}
$$

where $k_{i j}=\left|\vec{k}_{i}+\vec{k}_{j}\right|$. By adopting the $\delta N$ formalism, in the mixed modulated reheating scenario we have

$$
\tau_{N L}=\frac{R}{(1+R)^{3}}\left(\frac{N_{\sigma \sigma}}{N_{\phi}^{2}}\right)^{2},
$$

including the one-loop correction. From Eqs. (41) and (64),

$$
\tau_{\mathrm{NL}} \simeq\left(\frac{1+R}{R}\right) f_{\mathrm{NL}}^{2}
$$

Hence, for small $R$ there is a possibility of generating large $\tau_{\mathrm{NL}}$ which may be detected in the future experiments, without contradicting the current observational constraint on the isocurvature fluctuations. As discussed above, once we obtain a severe constraint on isocurvature fluctuations, the value of $f_{\mathrm{NL}}$ would be small in the mixed modulated reheating scenario. However, it is possible that a non-Gaussian signature comes from the trispectrum but not from the bispectrum. 


\section{$5 \quad$ Mixed curvaton scenario}

Now in this section, we consider CDM isocurvature fluctuations in the curvaton scenario. Isocurvature fluctuations in the curvaton have been investigated in the literatures [610]. Here we investigate this issue in the framework of a mixed scenario and focusing on how large non-Gaussianity can be produced without conflicting with the constraints on isocurvature fluctuations. Notice that the discussions given in this section apply not only to gravitino (axino) DM but also a generic CDM, although we focused on such DM candidates in the previous section. As pointed out in $[8,11]$, when the number density of CDM freezes before the curvaton decay, too large isocurvature fluctuations are generated and excluded by cosmological observations. However, this conclusion is valid for the original curvaton scenario in which fluctuations from the curvaton are only responsible for density fluctuations today. But, in general, fluctuations of the inflaton also contribute in addition to those from the curvaton. Such a mixed scenario has been extensively studied for the adiabatic fluctuations in [16-20] and for baryon isocurvature fluctuations [10]. Here we consider CDM isocurvature fluctuations in the mixed curvaton scenario for the cases with CDM being produced from the decay of the inflaton and/or the curvaton, paying particular attention to how large non-Gaussianity can be without conflicting with the isocurvature constraint.

In the same way as in the mixed modulated scenario, under the sudden decay approximation, the adiabatic curvature perturbations on the uniform (total) energy density hypersurface are analytically given by [20]

$$
\begin{aligned}
& \zeta=\zeta_{(\phi)}+\zeta_{(\sigma)} \\
& \zeta_{(\phi)}=N_{\phi} \delta \phi_{*}, \\
& \zeta_{(\sigma)}=N_{\sigma} \delta \sigma_{*}+N_{\sigma \sigma} \delta \sigma_{*}^{2}=\frac{2}{3} f_{\mathrm{dec}} \frac{\delta \sigma_{*}}{\sigma_{*}}+\frac{1}{3} f_{\mathrm{dec}}\left(1-\frac{4}{3} f_{\mathrm{dec}}-\frac{2}{3} f_{\mathrm{dec}}^{2}\right)\left(\frac{\delta \sigma_{*}}{\sigma_{*}}\right)^{2},
\end{aligned}
$$

where $\phi$ and $\sigma$ denote the inflaton and the curvaton, respectively, and $\sigma$ is taken to be positive without loss of generality \#9. We have neglected the non-linear part of fluctuations of the inflaton since they are very small. Here $f_{\text {dec }}$ is defined by

$$
f_{\mathrm{dec}}=\left.\frac{3 \rho_{\sigma}}{4 \rho_{r}+3 \rho_{\sigma}}\right|_{t=t_{\mathrm{dec}}},
$$

where $\rho_{\sigma}$ and $\rho_{r}$ are respectively energy densities of the curvaton field and radiation. $t=t_{\mathrm{dec}}$ is the time at the curvaton decay. For large non-Gaussianity, at least we need

$$
\frac{N_{\sigma \sigma}}{N_{\sigma}^{2}}=\frac{3}{4} \frac{1}{f_{\mathrm{dec}}}\left(1-\frac{4}{3} f_{\mathrm{dec}}-\frac{2}{3} f_{\mathrm{dec}}^{2}\right) \gg 1
$$

which leads to $f_{\text {dec }} \ll 1$. Hereinafter, we consider the case where this condition is satisfied.

\footnotetext{
\#9 The case where the second term dominates the linear term in $\zeta_{(\sigma)}$ corresponds to the "ungaussiton" scenario [32-34]. Our analysis includes such a scenario automatically.
} 
After the curvaton decay, the CDM isocurvature fluctuations are expressed as

$$
S_{\mathrm{CDM}}=3\left(\zeta_{\mathrm{CDM}}-\zeta\right),
$$

where $\zeta$ is given by Eq. (66) and $\zeta_{\mathrm{CDM}}$ is the curvature perturbation on the uniform CDM energy density hypersurface.

Regarding the production of CDM, one can consider two cases: dominant residual $\mathrm{CDM}$ is generated from the decay of the inflaton or the curvaton. In the following, we consider each case separately.

\subsection{Case with CDM from the inflaton decay}

First, let us consider the case where dominant residual CDM is generated from the decay of inflaton, which implies that CDM has the same fluctuations of the inflaton, that is, $\zeta_{\mathrm{CDM}}=\zeta_{\phi}$ which denotes the curvature perturbation on uniform inflaton energy density hypersurface. In the case where $f_{\text {dec }} \ll 1$, we can consider $\zeta_{\phi} \simeq \zeta_{(\phi)}$ and hence $\zeta_{\mathrm{CDM}} \simeq \zeta_{(\phi)}$. Then, we obtain $[20]$

$$
S_{\mathrm{CDM}}=-3\left(N_{\sigma} \delta \sigma_{*}+\frac{1}{2} N_{\sigma \sigma} \delta \sigma_{*}^{2}\right)=-3 \zeta_{(\sigma)} .
$$

Since we are considering a mixed scenario, the isocurvature fluctuations here should have uncorrelated and correlated parts with adiabatic fluctuations which originate from both the inflaton and the curvaton. With the definition of the correlation coefficient of Eq. (52), $\beta=1$ for this case. Then we separate isocurvature fluctuations into two parts as done in the previous section.

In Fig. 4, we plot contours of $f_{\mathrm{NL}}$ as well as $\alpha_{\text {corr }}$ (left panel) and $\alpha_{\text {uncorr }}$ (right panel) in the $\sigma_{*}-f_{\text {dec }}$ plane. The results are quite similar to those for the mixed modulated reheating scenario discussed in the previous section. At the current level of the constraint on isocurvature fluctuations, relatively large non-Gaussianity can be generated without conflicting the constraint. However, once the limit becomes severe as $\alpha_{\text {uncorr }}<0.01$ which is expected in PLANCK, $f_{\mathrm{NL}}$ should not be large in this case.

\subsection{Case with CDM from the curvaton decay}

Next, let us consider the case where dominant CDM component is generated from the decay of the curvaton, which implies that $\zeta_{\mathrm{CDM}}=\zeta_{\sigma}\left(\neq \zeta_{(\sigma)}=f_{\text {dec }} \zeta_{\sigma}\right)$ because $f_{\text {dec }} \ll 1$, where $\zeta_{\sigma}$ is the curvature perturbation on the uniform curvaton energy density hypersurface. Then, we can obtain $[20]$

$$
S_{\mathrm{CDM}}=\left(1-f_{\mathrm{dec}}\right)\left[2 \frac{\delta \sigma_{*}}{\sigma_{*}}-\left(1+2 f_{\mathrm{dec}}+\frac{2}{3} f_{\mathrm{dec}}^{2}\right)\left(\frac{\delta \sigma_{*}}{\sigma_{*}}\right)^{2}\right] .
$$



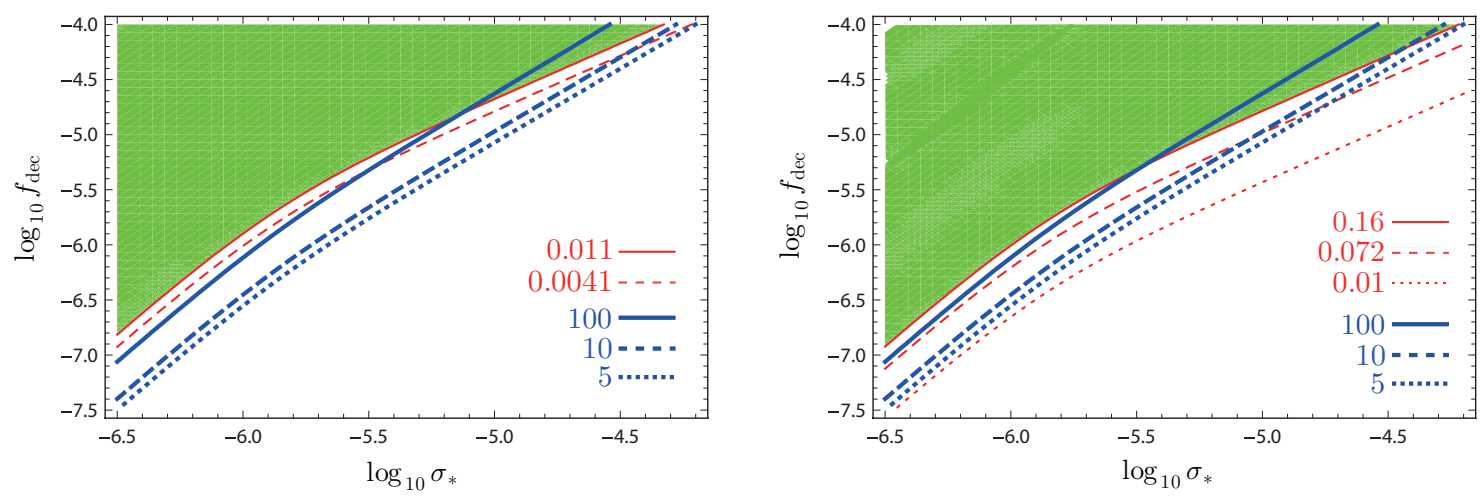

Figure 4: (color online) Contours of $f_{\mathrm{NL}}$ along with $\alpha_{\text {corr }}$ (left panel) and $\alpha_{\text {uncorr }}$ (right panel) in the $\sigma_{*}-f_{\text {dec }}$ plane. Red thin lines in the left panel show contours of $\alpha_{\text {corr }}=0.011$ (solid line) and 0.0041 (dashed line). In the right panel, we show contours of $\alpha_{\text {uncorr }}=$ 0.16 (solid line), 0.072 (dashed line) and 0.01 (dotted line). Green shaded regions are constrained by the current observational limit on isocurvature fractions from WMAP5 only. Blue thick lines are contours of $f_{\mathrm{NL}}=5$ (dotted line), 10 (dashed line) and 100 (solid line).

In the case where $f_{\mathrm{dec}} \ll 1, S_{\mathrm{CDM}}$ is approximately given by

$$
S_{\mathrm{CDM}} \simeq \frac{3}{f_{\mathrm{dec}}}\left[N_{\sigma} \delta \sigma_{*}-\frac{1}{2} N_{\sigma \sigma} \delta \sigma_{*}^{2}\right]
$$

with

$$
N_{\sigma}=\frac{2}{3} \frac{f_{\mathrm{dec}}}{\sigma_{*}}, \quad N_{\sigma \sigma}=\frac{2}{3} \frac{f_{\mathrm{dec}}}{\sigma_{*}^{2}}
$$

from Eq. (66). Then, the power spectrum of the isocurvature fluctuations is given by

$$
\begin{aligned}
P_{S_{\mathrm{CDM}}}(k) & \simeq 9 \frac{1}{f_{\mathrm{dec}}^{2}}\left[N_{\sigma}^{2}+N_{\sigma \sigma}^{2} \mathcal{P}_{\delta} \ln (k L)\right] P_{\delta}(k) \\
& =9 \frac{1}{f_{\mathrm{dec}}^{2}} P_{\sigma}(k) .
\end{aligned}
$$

In fact, in this case, we can easily find that CDM isocurvature fluctuations become large even for $f_{\mathrm{NL}}=O(1)$. From the above equation, the ratio of the power spectrum of the $\mathrm{CDM}$ isocurvature fluctuations to that of the adiabatic (curvature) perturbations is given by

$$
\frac{P_{S_{\mathrm{CDM}}}}{P_{\zeta}}=\frac{9}{f_{\mathrm{dec}}^{2}} \frac{P_{\sigma}}{P_{\zeta}}=\frac{9}{f_{\mathrm{dec}}^{2}} R
$$


From Eqs. (37), we obtain the following inequality:

$$
R \geq \frac{N_{\sigma}^{2}}{N_{\phi}^{2}}=\frac{8}{9} \epsilon \frac{f_{\mathrm{dec}}^{2}}{\sigma_{*}^{2}}
$$

where we have used $N_{\phi}^{2}=(2 \epsilon)^{-1}$ and Eq. (73) . Hence, we have the inequality given by

$$
\frac{P_{S_{\mathrm{CDM}}}}{P_{\zeta}} \geq 8 \frac{\epsilon}{\sigma_{*}^{2}}
$$

On the other hand, from Eqs. (41), assuming that $R \ll 1$, the non-linearity parameter $f_{\mathrm{NL}}$ can be written as,

$$
f_{\mathrm{NL}} \simeq R \frac{N_{\sigma \sigma}}{N_{\phi}^{2}}=\frac{4}{3} f_{\mathrm{dec}} R \frac{\epsilon}{\sigma_{*}^{2}} .
$$

Combining Eq. (78) with Eq. (77), we obtain

$$
\frac{P_{S_{\mathrm{CDM}}}}{P_{\zeta}} \geq R^{-1} f_{\mathrm{dec}}^{-1} f_{\mathrm{NL}}
$$

When $R \ll 1$ and $f_{\text {dec }}<1$, the fraction of the power spectrum of the CDM isocurvature fluctuations to that of the adiabatic (curvature) perturbations becomes much larger than the size of the non-Gaussianity, $P_{S_{\mathrm{CDM}}} / P_{\zeta} \gg f_{\mathrm{NL}}$. Thus, in the case where dominant CDM component is generated from the decay of curvaton, large non-Gaussianity from the (adiabatic) curvature fluctuations cannot be generated without conflicting observational constraint on CDM isocurvature fluctuations even at the current level.

\section{Non-Gaussianity from DM isocurvature fluctuations}

So far we have discussed non-Gaussianity of adiabatic fluctuations. However, isocurvature fluctuations can also produce large non-Gaussianity, which has been discussed recently in $[20,23,35,36]$. Thus in this section, we investigate non-Gaussianity from isocurvature fluctuations in our scenario.

First, we consider non-Gaussianity from isocurvature fluctuations in the modulated reheating scenario. As discussed in Sec. 4, the isocurvature fluctuations can be written, up to the second order, as

$$
S_{3 / 2}=\frac{1}{2} \frac{\Gamma^{\prime}}{\Gamma} \delta \sigma_{*}+\frac{1}{2}\left[\frac{1}{2} \frac{\Gamma^{\prime \prime}}{\Gamma}-\frac{1}{4}\left(\frac{\Gamma^{\prime}}{\Gamma}\right)^{2}\right] \delta \sigma_{*}^{2} .
$$

Depending on the explicit form of $\Gamma$ and the value of $\delta \sigma_{*}$, the discussion on non-Gaussianity can be divided into two cases. When the linear term dominates over the second order one in 
Eq. (80), it is convenient to define a non-linearity parameter for isocurvature fluctuations as in the case of adiabatic ones. We denote the non-linearity parameter as $f_{\mathrm{NL}}^{(\mathrm{iso})}$ and define it as the same as the counterpart in the adiabatic case:

$$
S_{3 / 2}=S_{g}+f_{\mathrm{NL}}^{(\mathrm{iso})} S_{g}^{2}
$$

where $S_{g}$ is the Gaussian part of isocurvature fluctuations. With this definition, $f_{\mathrm{NL}}^{(\mathrm{iso})}$ is calculated as

$$
f_{\mathrm{NL}}^{(\mathrm{iso})}=\frac{\Gamma^{\prime \prime} / \Gamma}{\left(\Gamma^{\prime} / \Gamma\right)^{2}}-\frac{1}{2}
$$

Notice that $f_{\mathrm{NL}}^{(\text {iso })}$ takes almost the same value as $f_{\mathrm{NL}}$ for adiabatic fluctuation although the sign is different. However, even if the size of $f_{\mathrm{NL}}^{(\mathrm{iso})}$ is comparable to that of the counterpart for the adiabatic mode, the signal from the bispectrum depends on the combination of $\alpha^{2} f_{\mathrm{NL}}^{\text {(iso) }}$ and $\alpha f_{\mathrm{NL}}^{\text {(iso) }}$ for the bispectrum coming from the 3-point function of $\langle S S S\rangle$ and $\langle S S \zeta\rangle$, i.e., purely isocurvature and correlated parts, respectively. Thus they are suppressed by the fraction of isocurvature perturbations $\alpha$, which is severely constrained by observations. Hence non-Gaussianity from isocurvature fluctuations cannot be large compared to the adiabatic one in this case.

However, when the second order term dominates over the first order one in Eq. (80), the argument becomes different. In this kind of case, the isocurvature fluctuations can be simply written as $S_{3 / 2}=S_{g}^{2}$, which is called the quadratic model in [23]. (The model characterized by Eq. (81) is called linear model in [23].) In the quadratic model, its power spectrum is determined by the second order term, thus non-Gaussianity in this model can be represented only with $\alpha_{3 / 2}$, which characterizes the size of the power spectrum of isocurvature fluctuations relative to the adiabatic ones and is given by

$$
\alpha_{3 / 2} \equiv \frac{P_{S_{3 / 2}}}{P_{\zeta}+P_{S_{3 / 2}}}
$$

This fraction should be small to fit to current data of CMB power spectrum, thus $P_{\zeta} \gg$ $P_{S_{3 / 2}}$ and using Eq. (49) and $R \ll 1$, we obtain the following relation,

$$
\alpha_{3 / 2}=\frac{P_{S_{3 / 2}}}{(1+R) P_{\phi}+P_{S_{3 / 2}}} \simeq \frac{1}{1+R} \frac{P_{S_{3 / 2}}}{P_{\phi}} \simeq \alpha_{\text {uncorr }}
$$

As discussed in the previous section, this parameter is constrained as $\alpha_{\text {uncorr }}<0.072$ at $95 \%$ C.L. Thus we use this value as a representative one in the following.

Here it should be noted that, even if we characterize the primordial non-Gaussianity for both adiabatic and isocurvature fluctuations with the nonlinearity parameters $f_{\mathrm{NL}}$ and $f_{\mathrm{NL}}^{\text {(iso) }}$, their evolutions to the present epoch, which are encoded in the transfer functions, are different. Thus the comparison of the non-linearity between isocurvature and adiabatic 
fluctuations is not so simple. However, some useful relations are obtained among $f_{\mathrm{NL}}, f_{\mathrm{NL}}^{\text {(iso) }}$ and $\alpha_{3 / 2}$ in [23]. Thus we make use of those relations.

Since non-Gaussianity from isocurvature fluctuations tends to be small for the case with the linear model, as discussed above, we here consider the quadratic case. Non-Gaussianity from the uncorrelated term can be estimated effectively as

$$
f_{\mathrm{NL}} \simeq 30\left(\frac{\alpha_{3 / 2}}{0.072}\right)^{1 / 2}
$$

where $f_{\mathrm{NL}}$ in the left hand side is the non-linearity parameter for adiabatic fluctuations. This relation has been obtained by finding $\alpha_{3 / 2}$ which gives the same $\mathrm{S} / \mathrm{N}$ for adiabatic $f_{\mathrm{NL}}$ assuming WMAP5 noise. As seen from the above relation, relatively large non-Gaussian fluctuations can be generated from isocurvature fluctuations even if we take the value allowed by current severe constraint on the isocurvature fluctuations.

Next we discuss non-Gaussianity from isocurvature fluctuations in the curvaton scenario. For the curvaton scenario, we considered two cases for the generation of CDM and discussed isocurvature fluctuations for each separetely. When CDM is created from the inflaton, isocurvature fluctuations are given by Eq. (70), from which we can evaluate the non-linearity parameter $f_{\mathrm{NL}}^{(\mathrm{iso})}$ as

$$
f_{\mathrm{NL}}^{\text {(iso) }}=-\left(\frac{1}{4 f_{\mathrm{dec}}}-\frac{1}{3}-\frac{f_{\mathrm{dec}}}{6}\right)=-\frac{1}{5} f_{\mathrm{NL}} .
$$

Notice that $f_{\mathrm{NL}}^{(\mathrm{iso})}$ is almost the same in size as the adiabatic counterpart $f_{\mathrm{NL}}$ except from the sign, which is similar to the case of the modulated reheating scenario. Although the size of the non-linearity parameters are almost the same between $f_{\mathrm{NL}}$ and $f_{\mathrm{NL}}^{(\mathrm{iso})}$, the signal of the bispectrum is suppressed by the isocurvature fraction as discussed above. Thus non-Gaussianity from the isocurvature fluctuations in this case would be also small as well.

The other case we considered is that CDM is produced from the decay of the curvaton. In this case, the isocurvature fluctuations are written as Eq. (71), then the nonlinearity parameter can be given by

$$
f_{\mathrm{NL}}^{(\mathrm{iso})}=-\frac{1}{4\left(1-f_{\mathrm{dec}}\right)}\left(1+2 f_{\mathrm{dec}}+\frac{2}{3} f_{\mathrm{dec}}^{2}\right) .
$$

As seen from this expression, when $f_{\text {dec }}$ is close to $1, f_{\mathrm{NL}}^{\text {(iso) }}$ would be very large. Thus in this case, even if the bispectrum itself is suppressed by the fraction of isocurvature fluctuations, its signal can be very large. However, notice that the sign of $f_{\mathrm{NL}}^{(\mathrm{iso})}$ is negative. In fact, when $f_{\text {dec }}$ is close to $1, f_{\mathrm{NL}}$ for the adiabatic fluctuations is $f_{\mathrm{NL}} \sim \mathcal{O}(1)$. Thus, when $f_{\text {dec }} \sim 1$, non-linearity mainly comes from isocurvature fluctuations and its size can be large but the sign is negative. Since too large value of $f_{\mathrm{NL}}^{\text {(iso) }}$ would be disfavored by observations, this scenario may contradict even with current observations. 
When the quadratic term dominated over the first order term, the argument is the same as the case with modulated reheating, in which the fraction of isocurvature fluctuation $\alpha$ gives the size of non-Gaussianity or bispectrum. Hence, when the quadratic term dominates in $\zeta_{(\sigma)}$, non-Gaussianity from isocurvature fluctuations can be large in the curvaton scenario as well.

\section{Summary and Discussion}

In this paper, we considered DM isocurvature fluctuations and non-Gaussianity in models where the adiabatic curvature fluctuations can be produced not only from of a light scalar field other than inflaton (modulus or curvaton), but from the inflaton fluctuations, which is called a mixed scenario. Regarding the non-Gaussianity of the curvature (adiabatic) fluctuations, we have found that relatively large non-Gaussianity can be realized as $f_{\mathrm{NL}} \sim$ $\mathcal{O}(10-100)$ without conflicting with the current constraints on the fraction of the CDM isocurvature fluctuations. In other words, the current limit on isocurvature fluctuations is not severe enough to prohibit large $f_{\mathrm{NL}}$ for such mixed scenarios. However, for the future CMB experiments such as Planck satellite, the limit on the uncorrelated isocurvature fluctuations will be improved as $\alpha_{\text {uncorr }} \lesssim 0.01$ [28-30]. We showed that this projected limit translates into the bound on the non-linearity parameter as $f_{\mathrm{NL}}<3$.

In fact, although we have mainly discussed non-Gaussianity in the curvature (adiabatic) fluctuations, nonlinearity can also arise from isocurvature fluctuations. We have also discussed non-Gaussianity of this type and found that it can be large as shown in Eq. (85), which corresponds to the size of the adiabatic nonlinearity parameter as $f_{\mathrm{NL}} \sim 10$ even with the projected Planck limit for $\alpha_{\text {uncorr }}$. However, it should be noted that the signature in the bispectrum of adiabatic and isocurvature fluctuations are not the same, thus we may differentiate non-Gaussianity from these fluctuations to some extent\#10. Thus, if we find that the value of the local type (adiabatic) non-linearity parameter is large in the future experiments, cosmological scenarios with gravitino DM may be disfavored even if we consider a mixed fluctuation scenario.

Furthermore, we have also investigated DM isocurvature fluctuations in the framework of the curvaton, in particular focusing on a mixed scenario. Our discussions for the curvaton case also apply to a generic DM, which originates from the inflaton or the curvaton. We have found that large non-Gaussianity in the curvature (adiabatic) perturbations is possible with the current level of isocurvature constraints in the curvaton case as well. However, as in the case of the modulated reheating scenario, if the limit becomes severer and large (adiabatic) non-Gaussianity of the local type is found in the future, DM are unlikely to be produced from the decay of the inflaton or the curvaton in the curvaton scenario, which would give important implications to the generation mechanism of DM.

\#10 The phase difference between adiabatic and isocurvature fluctuations in acoustic oscillations can help to distinguish non-Gaussinity from these two modes [37]. 


\section{Acknowledgments}

We thank Jun'ichi Yokoyama and Takahiro Tanaka for the collaboration at the early stage. We also grateful to Masahiro Kawasaki, Kazunori Kohri, and Fuminobu Takahashi for useful discussions. This work is supported by JSPS Grant-in-Aid for Scientific research, No. 19740145 (T.T.) and No. 21740187 (M.Y.). S.Y. is supported in part by Grant-in-Aid for Scientific Research on Priority Areas No. 467 "Probing the Dark Energy through an Extremely Wide and Deep Survey with Subaru Telescope". He also acknowledges the support from the Grand-in-Aid for the Global COE Program "Quest for Fundamental Principles in the Universe: from Particles to the Solar System and the Cosmos "from MEXT of Japan. We would like to thank the organizers of the IPMU workshop on "Focus week on non-Gaussianities in the sky" and the GCOE/YITP workshop YITP-W-09-01 on "Non-linear cosmological perturbations" for their hospitality, during which a part of this work was done.

\section{References}

[1] E. Komatsu et al. [WMAP Collaboration], Astrophys. J. Suppl. 180, 330 (2009) arXiv:0803.0547 [astro-ph]].

[2] K. M. Smith, L. Senatore and M. Zaldarriaga, arXiv:0901.2572 [astro-ph].

[3] S. Mollerach, Phys. Rev. D 42, 313 (1990); A. D. Linde and V. F. Mukhanov, Phys. Rev. D 56, 535 (1997) arXiv:astro-ph/9610219.

[4] K. Enqvist and M. S. Sloth, Nucl. Phys. B 626, 395 (2002) arXiv:hep-ph/0109214; D. H. Lyth and D. Wands, Phys. Lett. B 524, 5 (2002) arXiv:hep-ph/0110002 ; T. Moroi and T. Takahashi, Phys. Lett. B 522, 215 (2001) [Erratum-ibid. B 539, 303 (2002)] arXiv:hep-ph/0110096.

[5] G. Dvali, A. Gruzinov and M. Zaldarriaga, Phys. Rev. D 69, 023505 (2004) arXiv:astro-ph/0303591; L. Kofman, arXiv:astro-ph/0303614.

[6] T. Moroi and T. Takahashi, Phys. Rev. D 66, 063501 (2002) arXiv:hep-ph/0206026.

[7] D. H. Lyth, C. Ungarelli and D. Wands, Phys. Rev. D 67, 023503 (2003) arXiv:astro-ph/0208055.

[8] D. H. Lyth and D. Wands, Phys. Rev. D 68, 103516 (2003) arXiv:astro-ph/0306500.

[9] M. Beltran, Phys. Rev. D 78, 023530 (2008) [arXiv:0804.1097 [astro-ph]].

[10] T. Moroi and T. Takahashi, Phys. Lett. B 671, 339 (2009) arXiv:0810.0189 [hep-ph]].

[11] T. Takahashi, M. Yamaguchi, J. Yokoyama and S. Yokoyama, Phys. Lett. B 678, 15 (2009) arXiv:0905.0240 [astro-ph.CO]]. 
[12] M. Zaldarriaga, Phys. Rev. D 69, 043508 (2004) arXiv:astro-ph/0306006]; T. Suyama and M. Yamaguchi, Phys. Rev. D 77, 023505 (2008) [arXiv:0709.2545 [astro-ph]]; K. Ichikawa, T. Suyama, T. Takahashi and M. Yamaguchi, Phys. Rev. D 78, 063545 (2008) arXiv:0807.3988 [astro-ph]].

[13] N. Bartolo, S. Matarrese and A. Riotto, Phys. Rev. D 69, 043503 (2004) arXiv:hep-ph/0309033; K. Enqvist and S. Nurmi, JCAP 0510, 013 (2005) arXiv:astro-ph/0508573; K. A. Malik and D. H. Lyth, JCAP 0609, 008 (2006) arXiv:astro-ph/0604387]; M. Sasaki, J. Valiviita and D. Wands, Phys. Rev. D 74, 103003 (2006) [arXiv:astro-ph/0607627]; Q. G. Huang, arXiv:0801.0467 [hepth]; K. Ichikawa, T. Suyama, T. Takahashi and M. Yamaguchi, Phys. Rev. D 78, 023513 (2008) arXiv:0802.4138 [astro-ph]]; K. Enqvist and T. Takahashi, JCAP 0809, 012 (2008) arXiv:0807.3069 [astro-ph]]; Q. G. Huang, JCAP 0811, 005 (2008) arXiv:0808.1793 [hep-th]]; Q. G. Huang and Y. Wang, JCAP 0809, 025 (2008) arXiv:0808.1168 [hep-th]].

[14] K. Ichikawa, T. Suyama, T. Takahashi and M. Yamaguchi, in [12].

[15] G. Lazarides, R. R. de Austri and R. Trotta, Phys. Rev. D 70, 123527 (2004) arXiv:hep-ph/0409335.

[16] D. Langlois and F. Vernizzi, Phys. Rev. D 70, $063522 \quad$ (2004) arXiv:astro-ph/0403258.

[17] T. Moroi, T. Takahashi and Y. Toyoda, Phys. Rev. D 72, 023502 (2005) arXiv:hep-ph/0501007.

[18] T. Moroi and T. Takahashi, Phys. Rev. D 72, 023505 (2005) arXiv:astro-ph/0505339.

[19] K. Ichikawa, T. Suyama, T. Takahashi and M. Yamaguchi, in [13].

[20] D. Langlois, F. Vernizzi and D. Wands, JCAP 0812, 004 (2008) arXiv:0809.4646 [astro-ph]].

[21] A. A. Starobinsky, JETP Lett. 42 (1985) 152 [Pisma Zh. Eksp. Teor. Fiz. 42 (1985) 124]; M. Sasaki and E. D. Stewart, Prog. Theor. Phys. 95, 71 (1996); M. Sasaki and T. Tanaka, Prog. Theor. Phys. 99, 763 (1998).

[22] M. Sasaki, J. Valiviita and D. Wands, in [13].

[23] C. Hikage, K. Koyama, T. Matsubara, T. Takahashi and M. Yamaguchi, arXiv:0812.3500 [astro-ph].

[24] K. Rajagopal, M. S. Turner and F. Wilczek, Nucl. Phys. B 358, 447 (1991); L. Covi, L. Roszkowski and M. Small, JHEP 0207, 023 (2002) arXiv:hep-ph/0206119];

L. Covi, H. B. Kim, J. E. Kim and L. Roszkowski, JHEP 0105, 033 (2001) 
arXiv:hep-ph/0101009]; A. Brandenburg and F. D. Steffen, JCAP 0408, 008 (2004) arXiv:hep-ph/0405158.

[25] M. Kawasaki, K. Kohri, T. Moroi and A. Yotsuyanagi, Phys. Rev. D 78, 065011 (2008) arXiv:0804.3745 [hep-ph]].

[26] J. R. Ellis, J. E. Kim and D. V. Nanopoulos, Phys. Lett. B 145, 181 (1984); T. Moroi, H. Murayama and M. Yamaguchi, Phys. Lett. B 303, 289 (1993); M. Bolz, A. Brandenburg and W. Buchmuller, Nucl. Phys. B 606, 518 (2001) [Erratum-ibid. B 790, 336 (2008)] arXiv:hep-ph/0012052]; J. Pradler and F. D. Steffen, Phys. Rev. D 75, 023509 (2007) |arXiv:hep-ph/0608344].

[27] S. Nakamura and M. Yamaguchi, Phys. Lett. B 638, 389 (2006) arXiv:hep-ph/0602081]; T. Asaka, S. Nakamura and M. Yamaguchi, Phys. Rev. D 74, 023520 (2006) arXiv:hep-ph/0604132; M. Kawasaki, F. Takahashi and T. T. Yanagida, Phys. Lett. B 638, 8 (2006) arXiv:hep-ph/0603265]; Phys. Rev. D 74, 043519 (2006) arXiv:hep-ph/0605297]; M. Endo, K. Hamaguchi and F. Takahashi, Phys. Rev. Lett. 96, 211301 (2006) [arXiv:hep-ph/0602061]; M. Endo, F. Takahashi and T. T. Yanagida, Phys. Rev. D 76, 083509 (2007) arXiv:0706.0986 [hep-ph]].

[28] K. Enqvist and H. Kurki-Suonio, Phys. Rev. D 61, 043002 (2000) arXiv:astro-ph/9907221.

[29] M. Bucher, K. Moodley and N. Turok, Phys. Rev. D 66, 023528 (2002) arXiv:astro-ph/0007360].

[30] [Planck Collaboration], arXiv:astro-ph/0604069.

[31] C. T. Byrnes, M. Sasaki and D. Wands, Phys. Rev. D 74, 123519 (2006) arXiv:astro-ph/0611075.

[32] A. D. Linde and V. F. Mukhanov, in [3].

[33] L. Boubekeur and D. H. Lyth, Phys. Rev. D 73, 021301 (2006) arXiv:astro-ph/0504046].

[34] T. Suyama and F. Takahashi, JCAP 0809, 007 (2008) [arXiv:0804.0425 [astro-ph]].

[35] M. Kawasaki, K. Nakayama, T. Sekiguchi, T. Suyama and F. Takahashi, JCAP 0811, 019 (2008) arXiv:0808.0009 [astro-ph]].

[36] M. Kawasaki, K. Nakayama, T. Sekiguchi, T. Suyama and F. Takahashi, JCAP 0901, 042 (2009) arXiv:0810.0208 [astro-ph]].

[37] C. Hikage, D. Munshi, A. Heavens and P. Coles, arXiv:0907.0261 [astro-ph.CO]. 